Journal of Molecular Structure, 118 (1984) 47-52

Elsevier Science Publishers B.V., Amsterdam - Printed in The Netherlands

\title{
ELECTRON DIFFRACTION ANALYSIS OF CHARGE REDISTRIBUTION MODEL AND STRUCTURE OF SeF 6
}

\author{
LAWRENCE S. BARTELI a:d ANDING JIN
}

Department of Chemistry, The University of Michigan, Ann Arbor, MI 48109 (U.S.A.)

(Received 23 January 1984)

\begin{abstract}
The bond length of the octahedral molecule $S_{e} F_{6}$ was found to be $r_{g}(3 \sigma)=1.685(2) A$. Mean amplitudes of vibration, measured by diffraction, were within the experimental error of those calculated from spectral data by Brunvoll. Systematic residuals in scattered intensities were examined in the light of the observation (Pulay et al, Bartell et al.) that residuals for $\mathbf{S F}_{6}$ result principally from the disparity between the actial electron distribution and that of the independent atom model (IAM) of standard analyses. A modified version of IAM (MIAM), retaining spherical atoms as in LAM but shifting net charge and atomic radii, somewhat in the manner of Hehre et al, was tested. For reasons discussed, the MLAM approach worked too imperfectly to warrant routine incorporation in diffraction analyses.
\end{abstract}

\section{INTRODUCTION}

In a recent electron diffraction study of clusters of selenium hexafluoride formed by homogeneous nucleation in a supersonic jet $[1,2]$, it was desirable to know the precise internuclear distances and amplitudes of vibration. Therefore we undertook a diffraction investigation of $\mathrm{SeF}_{6}$ in the vapor phase because prio: structure determinations $[3,4]$ concluded a halfcentury ago were too rudimentary for our purposes. A secondary purpose for examining the substance was suggested by recent exhaustive studies [5-8] of $\mathrm{SF}_{6}$ : fairly large, reproducible intensity residuals remained even after the appreciable effect of dynamic scattering had been corrected. These residuals were traced to the difference between the actual electron distribution in the molecule and that implied by the independent atom model (IAM) used in the analysis $[6,9]$. While our investigation of $\mathrm{SeF}_{6}$ was under way, Hehre and co-workers $[10,11]$ showed that the outer-electron density contours of molecules can be reproduced quite satisfactorily by regarding molecules as superpositions of spherical atoms. Features of this approach which differed from those of the IAM of standard diffraction refinements included shifts in charge and characteristic radius of a given atom dependent upon the molecular environment. It seemed worthwhile to test whether a simple modification of the LAM with such shifts could provide a significant 
improvement in least-squares fits of experimental intensities. A preliminary investigation is reported in the following for $\mathrm{SF}_{6}$ as well as $\mathrm{SeF}_{6}$.

\section{EXPERIMENTAL PROCEDURE}

\section{Diffraction patterns}

A sample of $\mathrm{SeF}_{6}$ of purity in excess of $99.5 \%$ (NOAH Corporation) was used as received. Vapor at 180-200 Torr was admitted through a micronozzle, $0.12 \mathrm{~mm}$ in i.d., to the electron beam. Scattered intensities of 40.kV electrons were recorded on Kodak Medium Projector Slides through $r^{2}$ and $r^{3}$ sectors in an apparatus described elsewhere [12]. Asymmetry constants [13] $\hat{a}$ of $2 \AA^{-1}$ were adopted for the bonded and cis nonbonded distances, and of $1.1 \AA^{-1}$ for the trans distance. Shrinkages taken from Cyvin [14] were imposed, and intramolecular multiple scattering corrections [15] were introduced. Uncertainties, taking into account data correlation, were estimated as outlined in ref. 16 and 17. Experimental intensities are available from the authors upon a request accompanied by a self-addressed envelope.

\section{Treatment of charge distrikution}

It is assumed in conventional electron diffraction analyses, for simplicity, that molecular electron densities are the sum of Hartree-Fock atomic densities (the IAM approximation). We seek to find whether the incorporation of shifts in charge and atomic radius from free-atom values, such as those found in the work of Hehre et al. $[10,11]$ can profitably serve as the basis of a modified independent atom model (MIAM) appropriate for molecules. It is plausible to consider that the electron shifts occur only in the valence shells of the atoms and that these, for differential purposes, are adequately represented by a valence radial distribution $D_{v}(r)$ based on Slater-type orbitals [18], namely

$D_{\mathrm{y}}(r)=4 \pi n_{\mathrm{v}} N^{2} r^{2 n^{*}} \exp (-\alpha r)$

where $n_{V}$ is the number of electrons in the valence shell with orbital exponent $\zeta=(Z-\sigma) / n^{*} \equiv \alpha a_{0} / 2$, corresponding to effective principal quantum number $n^{*}$ and nuclear screening $\sigma$, and $N^{2}$ is a factor normalizing the integrated charge to $n_{r}$. Hehre's convention for the atomic radius, $r_{\mathrm{H}}$, is that the density $\rho\left(r_{\mathrm{H}}\right)=D\left(r_{\mathrm{H}}\right) / 4 \pi r_{\mathrm{H}}^{2}$ at $r_{\mathrm{H}}$ is 0.002 electrons $\AA^{-3}$. It is elementary to relate $r_{H}$ to exponent $\alpha$ and $n_{r}$ in eqn. (1).

Translating the shifts in $\alpha$ and $n_{v}$ to shifts in electron diffraction intensities, in turn, can be accomplished sufficiently well in the MIAM treatment through the approximation

$\left|f_{i}\right|_{\text {MIAM }}-\left|f_{i}\right|_{\text {IAM }}=-\left(2 / \sigma_{0} s^{2}\right)\left[\left(F_{i}\right)_{\text {MIAM }}-\left(F_{i}\right)_{\text {IAM }}\right]$ 
where $|f|$ is the absolute magnitude of the scattering factor for electrons and $F$ is the X-ray atom form factor. Equation (2), which is exact for the Born approximation, is a good approximation for the partial wave-scattering factors of $40-\mathrm{kV}$ electrons. Even if densities are not precisely expressible by the Slater distribution of eqn. (1), shifts in densities and in the scattering factor $\Delta F_{i}$ so calculated should be fairly good. Form factors for eqn. (2) are readily computed analy tically for the valence shell frox the integral

$F_{v}(\Delta)=n_{v} N^{2} \int_{0}^{\infty} 4 \pi r^{2 n^{*}} \exp (-\alpha r)(\sin s r) / s r d r$

\section{RESULTS}

Molecular parameters derived by conventional IAM analysis of the experimental reduced-intensity curve in Fig. 1 are compared in Table 1 with prior experimental and spectroscopic results. A correlation matrix is not given because off-diagonal elements were small.

Results of the MIAM treatment require comment, particularly in the degree to which a literal application of the Hehre model $[10,11]$ succeeds. Hehre and co-workers have calculated shifts in charge and radius for a series of sulfur compounds, including $\mathrm{SF}_{6}$ [19]. Results for $\mathrm{SeF}_{6}$ are not yet available but reasonable trial values can be tested. Ideally, if the MIAM treatment were a bona fide and accurate representation for hexafluorides, it would be expected that the loss $\Delta n_{\mathrm{v}}$ in the effective valence complement of the central atom would be gained by the fluorines. Further governing

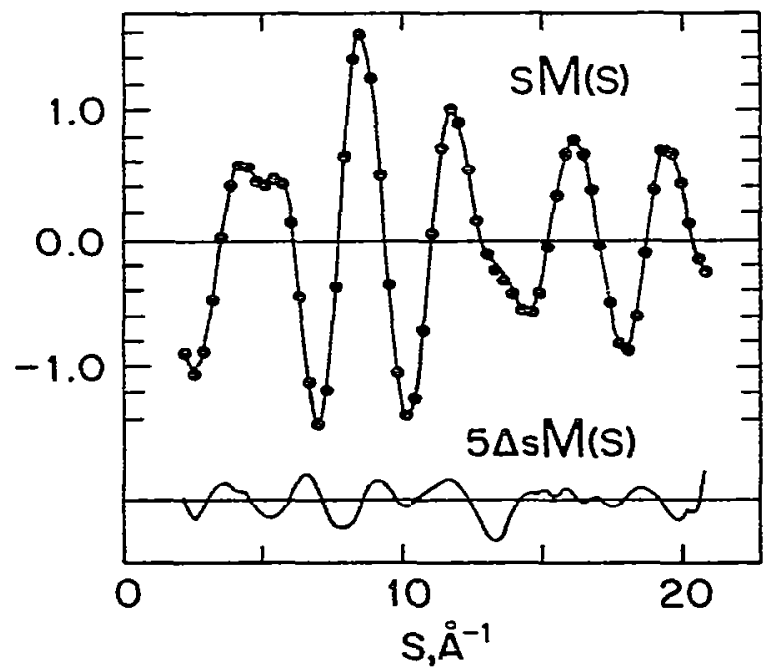

Fig. 1. Upper curve, reduced molecular intensity function sM(s) for selenium hexafuoride. $(\cdots)$, Experimental; ( $(-)$, calculated by IAM approximation. Lower curve, residuals $s M_{\exp }-s M_{\text {IAM, }}$ magnified five-fold. 
TABLE 1

Derived molecular parameters and estimated errors for $\mathrm{SeF}_{s}{ }^{\mathrm{a}}$

\begin{tabular}{llllll} 
& BPb & BK $^{\mathrm{c}}$ & ${ }_{\mathrm{g}}^{\mathrm{d}}$ & $l_{\mathrm{g}}^{\mathrm{d}}$ & $l_{\text {(spec) }}{ }^{\mathrm{e}}$ \\
\hline Se-F & $1.70(3)$ & $1.67(3)$ & $1.685(2)$ & $0.038(5)$ & 0.0399 \\
$\mathrm{~F} \cdot \mathrm{F} \mathrm{F}_{\text {cis }}$ & - & & $2.381^{\mathrm{f}}$ & $0.074(4)$ & 0.0717 \\
$\mathrm{~F} \cdot \mathrm{F} \mathrm{F}_{\text {trans }}$ & - & & $3.367^{\mathrm{f}}$ & $0.053(17)$ & 0.0533 \\
\hline
\end{tabular}

${ }^{a}$ Distance in $\&$. ${ }^{b}$ Brockway and Pauling [3]. ${ }^{c}$ Braune and Knoke [4]. d This research. Estimated uncertainties represent $3 \sigma$ and include random and suspected systematic errors and our estimate of effects of data correlation (due largely to errors intrinsic in the IAM treatment). ${ }^{\mathrm{e}} \mathrm{J}$. Brunvoll, as tabulated in ref. 14 . ${ }^{\mathrm{f}}$ Constrained to Se-F via shrinkages of ref. 14.

relations plausibly applicable to the present treatment are the strong correlations found by Hehre and co-workers [10,11] between atomic charge, atomic radius, and electronegativity. As will be discussed in the next section, the naive MIAM model, when constrained by such rational considerations, gave very poor results. Therefore, to see if the MIAM framework could yield rather better results if less restricted, we allowed the atoms in their molecular environments to take on arbitrary values of $n_{*}$ and $\alpha$. To illustrate the magnitude of effects we calculate difference functions

$[\Delta M(s)]_{\text {MIAM }}=M_{\text {MIAM }}-M_{\text {IAM }}$

and compare them with the experimental residuals $\Delta M_{\exp }(s)$, where $M(s)$ is the reduced intensity, $I_{\text {mol }} / I_{\text {at }}$. Resulis for selected computations are shown in Figs. 2 and 3.

\section{DISCUSSION}

The structural parameters in Table 1 are unexceptional. Their use in analyses of microcrystallites of $\mathrm{SeF}_{6}$ formed in supersonic nozzle flow, however, was helpful in determining structures of low-temperature phases nucleated by this procedure [1].

Results of the naive MIAM treatment do not engender optimism that the treatment will play a very useful role in electron diffraction. With reasonable parameters the $\Delta M_{\text {MIAM }}$ functions correlate better than randomly in magnitude and phase with experimental residuals. The results, then, suggest that in broad outline the model is correct even though in detail there are anomalies. Such a conclusion might have been anticipated from molecular orbital calculations of charge redistribution in diatomic molecules [20] and $\mathrm{SF}_{6}$ [9]. It is not just the surface distribution of Hehre and coworkers $[10,11]$ but also the inner, bonding distribution of electrons that shapes the potential function diffracting the electron beam which probes the molecular structure. A major improvement over the IAM treatment must take this interior electronic structure into account. 

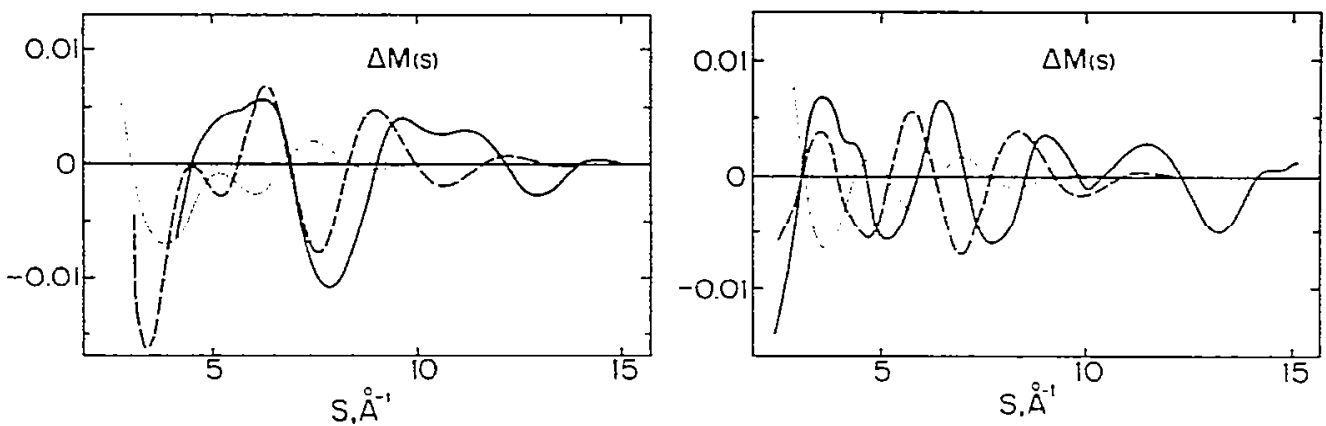

Fig. 2. (-), Experimental residuals $M_{\exp }-M_{\text {IAM }}$ for $\mathrm{SF}_{6} \cdot(\cdots)$, Residuals $M_{\mathrm{MIAM}}-$ $M_{\text {IAM }}$ calculated with atomic radii and charges similar to Hehre's [19]; MIAM $\alpha\left(n_{v}\right)$ parameters are $9.494(4.56)$ and $10.536(7.24)$ for sulfur and fluorine, respectively. $(---), \alpha\left(n_{v}\right)$ parameters are $8.42(6.00)$ and $9.8(8.00)$. Reference IAM $\alpha\left(n_{v}\right)$ parameters are $8.42(6.00)$ and $10.5(7.00)$. Units of $\alpha$ are $\AA^{-1}$.

Fig. 3. (-), Experimental residuals $M_{\text {exp }}-M_{\text {IAM }}$ for SeF $6 .(\cdots)$, Residuals $M_{\text {MIAM }}-$ $M_{\text {IAM, }}$, estimated to simulate Hehre's atomic radii and charges using STOs with $n *=4$, rather than the Slater rule value of 3.7 for Se; MIAM $\alpha\left(n_{v}\right)$ parameters are $8.6(4.56)$ and $10.536(7.24)$ for selenium and fluorine, respectively. (- - ) , $\alpha\left(n_{\mathrm{v}}\right)$ parameters are 7.7 (4.32) and 10.0 (7.28). Reference IAM $\alpha\left(n_{v}\right)$ parameters are $7.629(6.00)$, selected to fit Hartree-Fock $F_{v}(s)$ for Se with STO $n^{*}=4$; reference parameters are $10.5(7.00)$ for $\mathrm{F}$.

\section{ACKNOWLEDGEMENTS}

This research was supported by a grant from the National Science Foundation. We gratefully acknowledge a fellowship for one of us (A. J.) provided by the People's Republic of China. We thank Mr. John Stanton for carrying out computations and Dr. Edward Valente for assistance in several phases of work.

\section{REFERENCES}

1 E. J. Valente and L. S. Bartell, J. Chem. Phys., 79 (1983) 2683.

2 L. S. Bartell, E. J. Valente and R. Ramani, unpublished work.

3 L. O. Brockway and L. Pauling, Proc. Nati. Acad. Sci. (U.S.A.), 19 (1933) 68.

4 H. Braune and S. Knoke, Z. Phys. Chem., Abt. B, 21 (1933) 297.

5 L. S. Bartell and S. K. Doun, J. Mol. Struct., 43 (1978) 245.

6 L. S. Bartell, M. A. Kacner and S. R. Goates, J. Chem. Phys., 75 (1981) $2730,2736$.

7 S. R. Goates and L. S. Bartell, J. Chem. Phys., 77 (1982) 1866, 1874.

8 L. S. Bartell, M. A. Kacner and S. R. Goates, J. Chem. Phys., in press.

9 P. Pulay, R. Mawhorter, D. A. Kohl and M. Fink, J. Chem. Phys., 79 (1983) 185.

10 M. M. Francl, R. F. Hout, Jr. and W. J. Hehre, J. Am. Chem. Soc., 106 (1984) 563.

11 R. F. Hout, Jr. and W. J. Hehre, J. Am. Chem. Soc., 105 (1983) 3728.

12 L. S. Bartell, in A. Weissberger and B. W. Rossiter (Eds.), Physical Methods in Chemistry, 4th edn., Interscience, New York, NY, 1973.

13 L. S. Bartell, J. Mol. Struct., 84 (1982) 117.

14 S. J. Cyvin, Molecular Vibrations and Mean-Square Amplitudes, Elsevier, Amsterdam, 1968. 
15 B. R. Miller and L. S. Bartell, J. Chem. Phys, 72 (1980) 800.

16 L. S. Bartell and M. G. Anashkin, J. Mol. Struct., 17 (1973) 193.

17 R. A. Bonham and L. S. Bartell, J. Chem. Phys., 31 (1959) 702.

18 J. C. Slater, Phys. Rev., 36 (1930) 57.

19 W. J. Hehre, (1983) private communication.

20 D. A. Kohl and L. S. Bartell, J. Chem. Phys., 51 (1969) 2896. 